\title{
EL DERECHO COMO MODO DE COMPRENSIÓN FUNDAMENTAL EN EL PENSAMIENTO DE CARL SCHMITT ${ }^{1}$
}

\author{
Hugo E. Herrera \\ Instituto de Filosofía \\ Universidad Diego Portales \\ hugoeduardoherrera@udp.cl
}

\section{RESUMEN / ABSTRACT}

En el presente trabajo pretendo mostrar que el pensamiento de Carl Schmitt tiene un carácter marcadamente filosófico. Más precisamente: que en la base de él se halla una teoría de la comprensión. Esa teoría de la comprensión tiene la particularidad de que se desarrolla a partir de una teoría de la comprensión jurídica, pero se extiende, en su obra, a la comprensión en general.

Palabras Clave: Carl Schmitt, comprensión jurídica, hermenéutica, filosofía jurídica, juicio reflexionante, comprensión tecnológica.

\section{LAW AS A FUNDAMENTAL WAY OF UNDERSTANDING IN THE THOUGHT OF CARL SCHMITT}

In the present work, I intend to show that Carl Schmitt's thought is eminently philosophical, more precisely, that a theory of understanding lies at its ground. Peculiar to this theory is the fact that it is developed on the basis of a theory of juridical comprehension, although it extends, in his work, to human understanding in general.

KEYWORDS: Carl Schmitt, juridical understanding, hermeneutics, philosophy of law, reflective judgment, technological understanding.

\section{Autocomprensión de Schmitt como jurista}

Darl Schmitt es uno de los pensadores políticos y jurídicos más importantes del siglo XX. Su pensamiento ha sido interpretado de muy diversas maneras ${ }^{2}$. Usualmente es conocido por sus vínculos con el nacionalsocialismo, por su distinción política entre amigo y enemigo, por su doctrina de la excepción política y la soberanía,

\section{Este trabajo forma parte de un proyecto de investigación financiado por Fondecyt 1150102.}

Para un recuento, ver Hasso Hofmann 2002, pp. 1-2. 
sus contribuciones a la teoría del Estado y al derecho internacional. Recientemente, en el Handbook sobre él, editado por Jens Meierhenrich y Oliver Simons ${ }^{3}$, se distinguen tres aspectos de su pensamiento: uno político, uno jurídico y uno cultural. Son menos frecuentes, aunque relevantes, las interpretaciones que vinculan el pensamiento de Schmitt con la filosofía ${ }^{4}$.

En el presente trabajo pretendo mostrar que el pensamiento de Schmitt tiene un carácter eminentemente filosófico. Más precisamente: que en la base de él se halla una teoría de la comprensión. Esa teoría de la comprensión tiene la particularidad de que se desarrolla a partir de una teoría de la comprensión jurídica, pero se extiende, en su obra, a la comprensión en general.

Schmitt se entiende a sí mismo como jurista ${ }^{5}$. Se define de este modo en diversas obras $^{6}$. Tal descripción de su pensamiento importa ampliar el uso de las expresiones "jurista" y "jurisprudencia".

El derecho, plantea Schmitt, se ubica entre la tecnología y la teología ${ }^{7}$. El derecho queda incluido, así, en un esquema tripartito que abarca todas las posibilidades epistémicas disponibles. Pues, si a juicio de Schmitt, la tecnología se inclina hacia el polo de un "funcionalismo completo", la teología tiende, señala, hacia el extremo de un "completo substancialismo" (Schmitt 2015, p. 238). El derecho se ubica entre ambas, vale decir, ocupa una posición -comparativamente- equidistante respecto de los polos ideal y real de la comprensión. La tecnología apunta, entiende Schmitt, a constituirse en una racionalidad calculadora y controladora, en la cual el conocimiento es obtenido por la vía de la construcción del objeto o la subsunción de casos bajo reglas ${ }^{8}$. La teología, de su lado, está atada a un completo substancialismo en el sentido de que importa una completa entrega a una realidad trascendente ${ }^{9}$. En un caso se acentúa el polo ideal, en el otro, el real.

La posición intermedia que asume el derecho no debe ser entendida como un compromiso entre ambos extremos. Los textos muestran que para Schmitt la forma de comprensión jurídica se eleva por sobre las otras dos. El derecho como forma de comprensión queda caracterizado en el pensamiento de Schmitt, según veremos, porque él tematiza suficientemente la relación y la tensión entre los polos real y conceptual. La comprensión jurídica reconoce el polo real. Este polo emerge como develado y dotado de sentido, a la vez que como incontrolable e imprevisible. El derecho considera también, empero, las producciones de la mente, el polo ideal. Además, reconoce la heterogeneidad

Jens Meierhenrich y Oliver Simons 2016.

Ver, por ejemplo, Michael Marder 2010; Mika Ojakangas 2006; John P. McCormick 1997. Ver Schmitt 2015, p. 13.

Ver, por ejemplo, Schmitt 1996a, pp. 20-21; 1963, pp. 15-16; 1997, p. 6; 2015, pp. 13, 18, 100, 165, 237-38; 2002, p. 89; 2011, pp. 11, 32, 35; 1996b, p. 79, nota a pie de página 1. Ver Schmitt 2015, pp. 18, 100, 165, 237, 238; 2003, 420-422.

Ver Schmitt 2015, p. 121.

Ver Schmitt 2015, p. 238. 
de los polos, desde que, por una parte, las situaciones concretas emergen a partir de un trasfondo de misterio y eventual excepción, mientras que, de la otra, la comprensión intenta articular esas situaciones por medio de conceptos generales.

El problema de la tensión y la relación entre regla y caso aparece tempranamente como tema de consideración explícita por parte de Schmitt. También tempranamente revela alcances generales. En su obra no hay uno o más textos en los que se aborde el asunto de manera detallada y monográfica; no hay un texto dedicado exclusivamente a este tema. Sin embargo, existen múltiples pasajes repartidos por sus diferentes obras que, interpretados conjuntamente, sí permiten observar que Schmitt da un paso justificado al vincular la comprensión como tal y la comprensión jurídica, y al entender al derecho como forma fundamental de comprensión.

Lo que define a la comprensión y la reflexión jurídica y le otorga su carácter fundamental es la explícita tematización de las condiciones de la comprensión. En esto se diferencia la comprensión jurídica en sentido amplio de toda otra forma de comprensión y en esto consiste el que una forma fundamental de comprensión haya de ser llamada jurídica: en que tiene a la vista la relación y la tensión entre la regla y el caso, el concepto y la existencia, lo general y lo particular, la norma y la excepción.

En toda comprensión humana se opera dentro del contexto determinado por esos polos. Incluso toda comprensión queda puesta frente a la tarea de resolver dificultades prácticas generadas por la polaridad. Así, por ejemplo, se inventan y prueban nuevas reglas que vengan a dar mejor cuenta de sucesiones de fenómenos, o se ensayan usos del lenguaje que permitan expresar más pertinentemente una experiencia estética. Pero no en toda comprensión humana se eleva a asunto o tema de estudio y consideración la relación y la tensión de los polos de la comprensión. En el momento en el que algo así se efectúa, estamos, si seguimos a Schmitt, frente al pensamiento jurídico como pensamiento fundamental o a lo que denomina "filosofía de la vida concreta" (Schmitt 1996a, p. 21).

La posición superior que ocupa el derecho respecto de la teología y la tecnología es lo que justifica la inusual identificación que hace Schmitt de la comprensión jurídica y la comprensión humana en general, así como la que hace entre el pensamiento jurídico y la "filosofía de la vida concreta". La comprensión jurídica es capaz de abarcar la existencia incluyendo su "seriedad" y la tensión entre lo general y lo excepcional ${ }^{10}$. En "La situación de la jurisprudencia europea" el pensamiento jurídico es tenido por el modo de comprensión fundamental de la existencia. En este trabajo, Schmitt indica que los primeros grandes filósofos -Sócrates, Platón y Aristóteles-habrían sido "primariamente juristas y no lo que hoy son llamados filósofos". Continúa Schmitt especificando: "[N]aturalmente, con los términos jurista y jurisprudencia no entiendo algo así como un profesor que concibe su ciencia de acuerdo con la organización especializante de la docencia y la evaluación" (Schmitt 2003, p. 427). 
En los tres puntos siguientes analizaré, siguiendo las fechas de aparición, diversos textos de Schmitt. Trato de hacer patente cómo en ellos va emergiendo una teoría de la comprensión que, iniciándose en consideraciones específicamente jurídicas, se expande hacia la comprensión en general. En el punto final, intentaré dejar indicado, preliminarmente, aludiendo a tres autores relevantes, que los desarrollos teóricos de Schmitt tienen relevancia para la posterior teoría de la comprensión. Debido a que se trata de plantear una interpretación de carácter global del pensamiento schmittiano, es menester aclarar que el análisis de los textos se restringirá severamente al de pasajes escogidos que posean carácter decisivo para la hipótesis propuesta en el artículo.

\section{Implicancias del pensamiento jurídico en sentido usual para la comprensión en general}

El derecho en tanto que disciplina tiene a la vista, desde un inicio, como su problema, el de la relación y la tensión entre regla y caso. Schmitt plantea que, en la decisión judicial, el juez se encuentra enfrentado a la tensión entre normas generales y abstractas, de un lado, y un caso, singular y concreto, del otro. El caso es una situación que emerge desde un trasfondo real, heterogéneo con la normalidad ${ }^{11}$, develado en formas típicas ${ }^{12}$ y dotado de un cierto sentido ${ }^{13}$. Su hondura, conformación y sentido ponen al juez ante la exigencia de no someter simplemente a la situación, sino adecuarse a ella en su decisión ${ }^{14}$. Habrá situaciones que eventualmente pasarán fácilmente por los conceptos de la regla. Pero otras no ${ }^{15}$. El juez queda expuesto a un deber de justificación ${ }^{16}$. Ha de adoptar una decisión "correcta (richtige)" (Schmitt 2009, p. 1). Para alcanzarla, no puede mantenerse en el ámbito auto-contenido de las normas y sus significados, y simplemente sujetar, en su decisión, a los casos a esos significados normativos previos ${ }^{17}$. Un tal modo de operar importa un mero sometimiento de los casos, que desconoce su significado. Si la decisión ha de ser justa, el juez debe, en cambio, dar el paso "hacia otra esfera," a la "vida real (wirkliches Leben)" (Schmitt 2009, p. 28), a la "vida multiforme (vielgestaltige Leben)" (Schmitt 2009, p. 8) y considerar las "particularidades específicas (spezifische Besonderheiten)" (Schmitt 2009, p. 76) de las situaciones. La tensión entre regla y caso le deja puesto, entonces -aunque pueda eventualmente no hacerlo y aunque en muchos casos no lo haga-, frente a la exigencia de reflexionar sobre cómo ajustar

11 Ver Schmitt 2009, pp. 5, 8, 37, 76.

12 Ver Schmitt 2009, pp. 8, 76.

13 En virtud de ese sentido, que dota al caso "concreto" de una intensidad fundamentalmente distinta a la del caso simplemente pensado "como posible," la comprensión judicial posee el carácter de "praxis” (Schmitt 2009, pp. 58-9; véase, pp. 8, 28, 32, 41, 55, 76, 82, 96, 111-112; 1996a 18-19, 21; 1993, pp. 16-17, 19-20).

14 Ver Schmitt 2009, pp. 28, 32, 41, 59, 76, 93, 111-112.

15 Ver Schmitt 2009, pp. 37-8, 41, 68, 107.

16 Ver Schmitt 2009, p. 66; 1996a, p. 3.

17 Ver Schmitt 2009, p. 93. 
ambos de una forma plena de sentido. Deberá producir un fallo mediante una actividad de imaginación y reflexión, en el cual eventualmente mutará el significado de la regla ${ }^{18}$. Esto supone, al menos en sus rudimentos, desarrollar una atención respecto del distinto carácter de lo ideal y lo real, de las maneras en las que entran en relación y del hecho de que siempre persiste una tensión y heterogeneidad entre ambos ${ }^{19}$.

Schmitt comienza su derrotero intelectual prestándole atención a este problema específicamente jurídico en el sentido tradicional de la expresión, a saber, a la cuestión de la relación entre norma y caso, a propósito de la comprensión judicial, en Ley y juicio (Gesetz und Urteil), de donde extraigo las citas anteriores ${ }^{20}$. El asunto de la comprensión jurídica muestra, sin embargo, desde un inicio, alcances más amplios que los propios de la decisión judicial.

William Rasch ha mostrado plausiblemente que el "juicio" por medio del cual Schmitt intenta resolver el problema de la tensión entre regla y caso, "posee ... una asombrosa semejanza con aspectos del juicio estético kantiano" (Rasch 2004, p. 100). Ante el problema que plantea la heterogeneidad de las reglas y los casos, y el sentido con el que éstos emergen -detectados por Schmitt en la situación de la comprensión judicial, por Kant en la situación de la comprensión estética-, la comprensión tiene vedado el camino de la subsunción determinante. Schmitt rechaza la idea de que la corrección de la decisión judicial pueda definirse por su "conformidad a la ley (Gesetzmäßigkeit)," considerada la ley como "norma" con un "significado establecido de manera previa (en un sentido temporal y lógico) a la decisión" (Schmitt 2009, p. 93). Si la comprensión ha de ser adecuada o correcta, ella debe dar el paso desde el orden de las reglas hacia la dimensión real de lo concreto, por medio de un "juicio reflexivo" (Rasch 2004, p. $100)^{21}$. El juez -señala Schmitt- ha de producir "una decisión general para el caso concreto" (Schmitt 2009, p. 93). De manera parecida a como en el juicio estético, el juez puede esperar que los "fundamentos de su decisión conduzcan a una persuasión general" (Schmitt 2009, p. 93), vale decir, al reconocimiento de la corrección del fallo por parte de una comunidad ${ }^{22}$. Esta pretensión es justificable a partir de la circunstancia de que la decisión del juez busca ser correcta teniendo a la vista cómo otros decidirían:

Ver Schmitt 2009, pp. 28, 32, 93-4, 111-112.

Ver Schmitt 2009, pp. 5-9.

Sobre la comprensión de Schmitt del derecho y la decisión en sus escritos tempranos, ver, por ejemplo, Paul Oertmann, 1913, pp. 817-818; Felix Halldack, 1912, pp. 464-467; Walsmann, 1913, pp. 431-432; Walter Jellinek, 1914, pp. 296-299; H. Hofmann, 2002, pp. 25-37; L. Kiefer, 1990, pp. 480-482; Ulrich Habfast, 2010, pp. 8-36; Gary Ulmen, 1991, pp. 102-103; V. Neumann, 2015, pp. 20-1; William Scheuerman, 1999, pp. 4, 19-20; A. Adam, 1992, p. 56; Ingeborg Maus, 1980, pp. 86-88; John P. McCormick, 1997, pp. 21112; 1999, pp. 413-428; Carlo Galli, 1996, pp. 313-331; Heiner Bielefeldt, 1994, pp. 20-3. Ver Schmitt 2009, pp. 28, 32, 59, 112, etc.

Ver I. Kant 1968, §§ 39-41. Sobre la relevancia del juicio estético en la comprensión política, véase H. Arendt 1992, pp. 65-77. 
la decisión jurídica es "correcta cuando uno puede asumir que otro juez hubiese decidido de la misma manera (ebenso entschieden hätte)" (Schmitt 2009, p. 68).

A partir de estas consideraciones, el libro de Schmitt queda situado en un contexto más amplio. La forma de comprensión jurídica schmittiana puede ser considerada como adecuada no solo para el campo estrictamente judicial. Ella se realiza de modo similar a la comprensión estética. Según veremos a continuación, Schmitt pondrá en cuestión tempranamente la filosofía teorética kantiana, por soslayar el aspecto concreto de la existencia, a partir del énfasis puesto en las "formas objetivamente válidas" del "pensamiento" (Schmitt 1998, p. 63). Esta crítica se enmarca dentro de una consideración general sobre los límites del racionalismo, por parte del pensamiento de lo excepcional de Schmitt. La forma de comprensión jurídica, en la medida en que tematiza la tensión y la relación entre regla y caso, sin soslayar el carácter concreto y peculiar del caso, muestra su capacidad para volverse la manera de abordar todas aquellas situaciones y ámbitos en los que no sea admisible clausurar de antemano la hondura, indeterminación y sentido que los afectan, por la vía de la reducción del caso a caso-de-regla. Vale decir, la forma de comprensión jurídica revela su aptitud para convertirse en modo de comprensión fundamental de la existencia.

\section{Del pensamiento jurídico en sentido estricto o usual al pensamiento jurídico como comprensión fundamental}

Cuatro años después de Ley y juicio, en Theodor Däublers "Nordlicht", Schmitt hace el tránsito explícitamente desde el campo específicamente jurídico hacia la existencia en su sentido más amplio. Aborda otra vez el problema de la relación y la tensión entre regla y caso, pero ahora como la cuestión de la comprensión en general. Este texto es un comentario de un poema épico. En él, Schmitt repara en que la existencia humana emerge desde un trasfondo de insondabilidad y misterio, tanto por el lado de la realidad cuanto por el del sujeto ${ }^{23}$. La existencia surge desde "[1]as profundidades del mundo y el alma" (Schmitt 1991, p. 51). La actividad de comprensión de esa existencia se articula en una "intuición que se vuelve palabra" (Schmitt 1991, p. 51). La comprensión se desenvuelve entre dos polos: por un lado, uno existencial, a la vez develado e insondable; por otro lado, el de las "palabras" y "formulaciones" con las que el sujeto lleva la existencia al pensamiento articulado (Schmitt 1991, pp. 51, 66). Esta polaridad puede llegar a ser concebida como un "dualismo" (Schmitt 1991, pp. $31,63,70$ ) de lo ideal y lo real. Schmitt se distancia de esta posición. En cambio, entiende a los polos ideal y real como partes de una relación y una tensión, como una cierta unidad de opuestos en tensión ${ }^{24}$.

23 Esta idea será persistente en el pensamiento de Schmitt; ver 1996a, p. 15; 2015, pp. 64, 388.

24 Ver Schmitt 1991, pp. 52, 55. 
A partir del reconocimiento de la relación y la tensión entre los polos ideal y real, se abren diversos caminos para la comprensión. En los extremos se encuentran la "negación inactiva" (Schmitt 1991, p. 57) y la manipulación tecnológica (la "astuta técnica de la época mecanicista", Schmitt 1991, p. 56). En el primer caso puede hablarse de una entrega pasiva al polo real; en el segundo, de una inclinación hacia el polo ideal de las producciones de la mente. Entre ambos extremos, Schmitt admite una comprensión que, sin abandonarse al polo real, busca lograr una articulación mental de la existencia que no caiga, empero, en el extremo tecnológico o funcionalista. Este tipo de comprensión se distingue porque en él se intenta captar la hondura existencial de lo real para conducirla a las expresiones y articulaciones mentales de la palabra y el pensamiento ${ }^{25}$. El lenguaje puede volverse "el contenedor [Gefä $\beta$ ]" de esa hondura existencial, la "intuición" ser llevada a la "palabra", la palabra "descubrir lo que aloja de conocimiento" y ese conocimiento expresarse en "formulaciones filosóficas" (Schmitt 1991, p. 51).

La consideración de la tensión y la relación de los polos de la comprensión, y de la tecnología como un modo de comprensión que se inclina al funcionalismo, le permiten a Schmitt efectuar ya aquí una temprana crítica, que prefigura la que más tarde hará en "La época de las neutralizaciones y despolitizaciones", de la posición, que asume, a su juicio, Max Weber y su generación. Ellos admiten una "oposición de mecánica y espíritu" (Schmitt 1991, p. 63). Sin embargo, no repararon en que la técnica no es la mera "mecánica", en que tras la mecánica hay algo así como un "espíritu" (Schmitt 1991, p. 66); vale decir, que ella es expresión de una forma de comprensión que se inclina hacia el polo funcional. A diferencia de esta posición, Schmitt sí atiende a lo que podría llamarse el trasfondo que anima la maquinaria: al "espíritu de la época mecanicista", esto es, una forma de comprensión en la cual "el entendimiento se libera de todas las cadenas y sigue sin impedimentos su racionalismo; su fin es conocer la tierra para dominarla" (Schmitt 1991, p. 66) ${ }^{26}$.

Pocos años más tarde, en Romanticismo político, Schmitt aborda nuevamente el problema de la relación y la tensión entre regla y caso como la cuestión de la comprensión jurídica y de la comprensión en general. Con la modernidad aparece un dualismo, expresado de manera filosóficamente nítida en la separación cartesiana de pensamiento y realidad. Con esa separación se vuelve problemático el acceso de la mente a un "ser real", que deviene irracional e inexplicable (Schmitt 1998, p. 52). Entonces, la cuestión de la comprensión emerge y resulta formulable de manera fundamental, en los siguientes términos: ¿cómo puede ocurrir que reglas -causales, jurídicas o morales-, los conceptos, pensamientos, la mente, lleguen a comprender lo que en principio es heterogéneo con ellos, a saber, el ser, la realidad, los casos?

$25 \quad$ Ver Schmitt 1991, pp. 47, 51.

26 J. P. McCormick repara en las semejanzas entre esta posición tempranamente adoptada por Schmitt y la que más tarde asume Martin Heidegger; véase J. P. McCormick 1997, pp. 45, 97; M. Heidegger 2000, p. 27. 
Frente a esta pregunta, el romanticismo toma el camino hacia la realidad y su sentido. Schmitt lo entiende como un movimiento que, en reacción al racionalismo, se inclina hacia una estetización de la existencia, privilegiando el polo real sobre el conceptual $^{27}$. Él repara en las insuficiencias de la posición romanticista, a partir de la consideración de las condiciones de la comprensión. Con su énfasis en lo real y su soslayo de lo conceptual, el romanticismo acaba renunciando a una "relación adecuada con el mundo visible externo" (Schmitt 1998, p. 93), con la "realidad concreta" (Schmitt 1998, p. 20). La falta de distanciamiento reflexivo y racional le hace, al romanticismo, perder capacidad para comprender la realidad allende el enunciado de notas ocasionales o caprichosas ${ }^{28}$.

Schmitt se distancia, empero, también de lo que entiende como una vía de solución funcionalista o racionalista al dualismo, expresada en la filosofía teórica de Immanuel Kant. Schmitt señala que Kant falla en su intento de superación del dualismo porque acaba aislando al pensamiento respecto de la existencia. En la teoría kantiana, la existencia termina siendo reemplazada por la "objetividad". Esta "consiste en que el pensamiento se mueve en formas objetivamente válidas (darin besteht, dass das Denken sich in objektiv gültigen Formen bewegt)" (Schmitt 1998, p. 63). Queda, entonces, de un lado el pensamiento y su objetividad, y, del otro, una situación existencial que, debido a su hondura y sentido, no resulta objetivable, a la que tal pensamiento objetivante carece, en consecuencia, de paso.

En Teología política, Schmitt regresa otra vez sobre el problema jurídico de la tensión y la relación entre la regla y el caso. Se trata nuevamente de una cuestión de alcance, a la vez, jurídico y filosófico, la que se inscribe ahora dentro de la relación y la tensión de "lo general" y "la excepción" (Schmitt 1996a, p. 21).

La excepción afecta tanto a la "jurisprudencia” cuanto a la comprensión filosófica. Así como dentro de la "teoría positiva del Estado" y en la teoría del derecho kantiana y neokantiana, la excepción queda excluida de antemano, algo similar ocurre dentro del racionalismo teórico. "Sería un racionalismo consecuente decir que la excepción no prueba nada y que sólo lo normal puede ser el objeto de interés científico. La excepción confunde la unidad y el orden del esquema racionalista" (Schmitt 1996a, pp. 18-20). En cambio, para una "jurisprudencia" que se salga de los límites del normativismo y el racionalismo, "[1]a excepción persiste, empero, accesible” (Schmitt 1996a, pp. 1819). También para una comprensión -jurídica- general o lo que Schmitt designa como "filosofía de la vida concreta" la cual "no debe evadirse de la excepción ... sino que debe estar interesada en ella en el grado más alto" (Schmitt 1996a, p. 21).

Es desde una comprensión que incluya la consideración de la excepción, que, a juicio de Schmitt, recién se puede alcanzar consciencia respecto de los alcances y límites de la normalidad, las reglas y conceptos. Hemos visto que Schmitt entiende, en Romanticismo político, que el racionalismo kantiano falla en su explicación sobre 
la manera de superar el dualismo entre lo ideal y lo real. Esta insuficiencia se debe, en último término, a algo que aparece con mayor claridad a partir del pensamiento de lo excepcional, en Teología política. Al poner el énfasis en las construcciones de la mente, aquella posición soslaya la consideración del polo opuesto a las construcciones: la excepción, el trasfondo oculto e incontrolable desde el cual la existencia emerge ${ }^{29}$.

La "filosofía de la vida concreta" realiza una tematización de la existencia como excepcional y, a la vez que oculta, develada y dotada de un sentido ${ }^{30}$. Ese pensamiento mantiene a la vista los dos polos, el excepcional-existencial y el conceptual. Desde esa atención, realiza una conceptualización que, sin abandonarse al polo real, repara, empero, en su carácter -aunque, en tanto develado, discernible-, en último término, también insondable ${ }^{31}$. Dada la heterogeneidad de los términos de la comprensión y el sentido con el que se devela la existencia, la tarea comprensiva no puede consistir en una simple entrega del sujeto al polo real (como en el romanticismo), ni en una subsunción de lo dado bajo conceptos y reglas cuyo significado quede inalterado. Provisto con las reglas y conceptos mediante los cuales realiza la comprensión, quien comprende ha de dar el paso desde la esfera ideal de las reglas y conceptos hacia la esfera real de los casos. Entonces puede percatarse de la eventual diferencia del significado de las reglas y conceptos, de un lado, y el sentido concreto de la situación, del otro. Puesto ante esa tensión, quien comprende, sin saltarse simplemente las reglas y conceptos, ha de mediar entre ambos sentidos y, cuando sea exigible, adecuar el significado de la regla al significado concreto de la situación.

\section{El pensamiento schmittiano maduro}

En El concepto de lo político, Schmitt repara nuevamente en la cuestión de la tensión y la relación entre la regla y el caso como problema general de la comprensión. Distingue la esfera ideal de los conceptos y la de lo real. Plantea que la comprensión, si ha de ser correcta, exige dar el paso desde la esfera ideal a la real. Solo bajo condición de ese paso se halla, quien comprende, en condiciones de "reconocer, comprender y juzgar correctamente la situación concreta” (Schmitt 1963, p. 27). El resultado de esta actividad comprensiva puede ser, entonces, un concepto "existencial". Se trata de una noción nutrida del sentido y la hondura de lo real. El desconocimiento, en cambio, de esta exigencia de paso a la "realidad existencial" (Schmitt 1963, p. 28), culmina en un pensamiento autocontenido que persiste en las "abstracciones" e "ideales normativos" (Schmitt 1963, p. 28).

En la segunda parte de El concepto de lo político, "La época de las neutralizaciones y despolitizaciones", Schmitt indica: "todos los conceptos del ámbito espiritual”-vale decir, no solo las nociones jurídicas en sentido estricto, sino los conceptos prácticos y 
los teóricos- "son pluralistas en sí mismos" (Schmitt 1963, p. 84) 32. También señala: "Nosotros reconocemos el pluralismo de la vida del espíritu" (Schmitt 1963, pp. 9495). A continuación del primer pasaje agrega: "Todos los conceptos fundamentales no son normativos, sino existenciales" (Schmitt 1963, p. 84). Afirma, asimismo, que en "todo concepto y palabra" existe una irreductible "ambigüedad" (Schmitt 1963, p. 84). Todas estas frases apuntan a tematizar, desde una posición jurídica en sentido amplio o filosófica, la comprensión y sus condiciones, en especial, la tensión que existe entre los conceptos y la existencia concreta. La consciencia respecto de la "ambigüedad" de los conceptos y palabras emerge cuando se repara en la diferencia entre ellos, considerados con independencia de la existencia concreta, y esa existencia concreta. Previo a la aplicación, el concepto está aún indeterminado. Es, entonces, ambiguo: puede dar base a distintos modos de comprensión. Al ser aplicado, su contenido cambia, se determina, en la medida en que ha de ser reinterpretado desde el sentido al que se accede en la existencia concreta. El carácter "pluralista" de los conceptos, al que alude Schmitt, es, entonces, irremontable, pues en cada acto comprensivo, si se considera el sentido de la existencia concreta, el concepto reinterpretado adquiere un nuevo significado. La comprensión queda persistentemente bajo la exigencia de atender a la situación y su hondura existencial. "Todos los conceptos fundamentales no son normativos sino existenciales" quiere decir que -si los conceptos han de ser algo distinto a fórmulas abstractas, con las que simplemente se someta a la realidad- la comprensión requiere ir desde la fórmula hacia la existencia concreta y desde allí reinterpretar la regla. A este tipo de comprensión -jurídico, atento a la existencia concreta- Schmitt lo llama allí "una comprensión integral", a saber, una que no enfatiza solo las reglas y considera todos los aspectos involucrados en la comprensión (Schmitt 1963, p. 95).

En Sobre los tres modos de pensar la ciencia jurídica, Schmitt subraya la similitud entre "inteligencia (Intelligenz)" y la "jurisprudencia" (Schmitt 1993, p. 34). Ambas operan de una manera similar. Toda la comprensión acontece basada en un contexto previo de conceptos y reglas ${ }^{33}$. En la comprensión, por medio de reglas y conceptos, el sujeto intenta hacer luz sobre los casos. La comprensión adecuada de los casos exige atender a los contextos situacionales y al sentido presente en ellos, lo que, nuevamente, lleva a Schmitt a advertir contra los esfuerzos por simplemente reducir las situaciones a las reglas ${ }^{34}$. Schmitt critica, en este sentido, al "funcionalismo" (Schmitt 1993, pp. 17, 34). Éste, debido al énfasis puesto en el polo conceptual y las reglas, pierde capacidad comprensiva respecto de la existencia concreta, de su "orden" y su eventual "desorden" (Schmitt 1993, pp. 15-17). Asimismo, no toma en consideración que la develación de la existencia como "orden" es condición de la validez de las reglas, así como de la comprensión en general. Ni las reglas de la "jurisprudencia", ni las de la "inteligencia" podrían tener aplicación sobre una situación que no se develara de maneras típicas y

Ver Schmitt 2014, p. 162.

Ver Schmitt 1993, pp. 16-20.

Ver Schmitt 1993, pp. 11-20, 34. 
dotada de un significado discernible, no radicalmente heterogéneo con los significados de las reglas (Schmitt 1993, p. 34)

En la nota que Schmitt añade en 1958 al artículo "La situación de la ciencia jurídica europea", afirma: "La filosofía del derecho no es, para mí, un vocabulario contenido en un sistema filosófico ya existente y recién luego aplicado sobre asuntos jurídicos, sino que el despliegue de conceptos concretos emergentes desde la inmanencia de un orden jurídico y social concreto". Un tal "pensamiento jurídico", agrega, "liberado de los callejones sin salida de los conceptos generales" es "superior a toda filosofía" (Schmitt 2003, p. 427). El derecho, en este sentido amplio, es el modo fundamental de comprensión. Él puede, sin embargo, inclinarse al polo funcionalista, entrar en "el callejón sin salida de los conceptos generales" o, en cambio, elevarse, en un gesto propiamente jurídico, a una comprensión lúcida respecto de la tensión y la relación entre regla y caso. Esa lucidez impone la exigencia de, en el acto comprensivo, considerar la dimensión real de lo concreto y su sentido existencial, para, desde allí, y recogiendo ese sentido de la situación, llevarlo al concepto ${ }^{36}$. Esta dimensión concreta no puede ser algo caótico o compacto, sino que ha de emerger de antemano como discernible ${ }^{37}$.

La tiranía de los valores contiene, en palabras de Schmitt, "las reflexiones de un jurista sobre la filosofía de los valores" (Schmitt 2011, pp. 32, 35). Schmitt se refiere, allí, en tanto que jurista, a un asunto filosófico. Está entendiendo al derecho en su sentido amplio. La crítica "jurídica" pretende coincidir con la de Martin Heidegger, a quien Schmitt cita extensamente ${ }^{38}$. El rendimiento de una consideración "jurídica" puede ser filosófico, siempre que, como ya ha indicado Schmitt en "La situación de la ciencia jurídica europea", no se restrinja las nociones de filosofía y derecho según la división usual de los saberes ${ }^{39}$. El problema de los valores es jurídico en sentido amplio, en la medida en que la filosofía de los valores viene a ser expresión de una reacción a la situación existencial en la que el "cientificismo de la naturaleza [Naturwissenschaftlichkeit]" coloca al ser humano y a las "ciencias del espíritu [Geisteswissenschaften]". El énfasis de la nueva racionalidad científica en un objeto neutralizado condujo a buscar en los

35 Ver Schmitt 1993, pp. 16-19.

$36 \quad$ Ver Schmitt 2003, p. 427.

$37 \quad$ Ver Schmitt 2003, p. 411; 1996a, p. 19; 1993, pp. 16-20; 1997, p. 6.

38 Ver Schmitt 2011, pp. 31, 38. En su comentario a la nueva edición del texto, Christoph Schönberger repara en la auto-comprensión de Schmitt como jurista: entre la teología y la tecnología; limita, empero, los alcances de la consideración jurídica schmittiana a los contornos del derecho tal como es usualmente entendida la disciplina. Por eso, Schönberger se desconcierta y se pregunta “¿A qué nivel se mueve realmente la argumentación de Schmitt? ... Su texto escoge desde un inicio una difícil posición intermedia entre filosofía y jurisprudencia”. C. Schönberger, 2011, p. 58. Una vez que se ha reparado, en cambio, en las reflexiones de Schmitt sobre los polos de la comprensión y las posiciones comprensivas admisibles, el derecho adquiere un alcance eventualmente mayor que el de la disciplina en sentido usual y adquiere pleno significado una crítica jurídica a los modos de comprensión racionalizantes o estetizantes. 
"valores" un "sustituto" al sentido de la existencia que procuran comprender las "ciencias del espíritu" (Schmitt 2011, pp. 24, 29-30, 38). La filosofía de los valores, sin embargo, dificulta una comprensión correcta o no abusiva de la situación. Ella se inclina hacia la consideración del sentido del polo ideal, soslayando el significado de lo real ${ }^{40}$. La "posición (Setzung)" de un valor importa -por la "lógica específica" del "valor"- la disvaloración de lo que se le opone (Schmitt 2011, 48.51). "La exigencia de hacer prevalecer los valores se vuelve en la coerción de imponer los valores directamente" (Schmitt 2011, 52). La ponderación atenta del significado ideal de los conceptos en conjunto con el significado real de las situaciones concretas, la mediación entre el significado del ideal y el de una existencia que emerge originariamente dotada de sentido, cede paso a una disputa de posiciones valorativas que pujan por la ejecución inmediata del ideal.

\section{Relevancia de la expansión del modo de comprensión jurídico}

El derecho, entonces, entendido en los textos de Schmitt como forma fundamental de comprensión, se caracteriza por realizar una tematización de la tensión y la relación entre la situación y las reglas y conceptos, el significado de unos y otros, tensión, relación y significados que están en la base de la comprensión humana en general. Existe en tanto modo fundamental de comprensión mientras no se deja de prestar consideración a los dos polos de la tensión y a la manera en la que una tal relación es posible.

La interpretación que he propuesto permite develar la contribución del pensamiento jurídico de Schmitt, tomado en sentido amplio, a una teoría de la comprensión humana. Schmitt logra dar, según hemos visto, durante la década del 10 y comienzos de la del 20, un paso que alcanzará relevancia decisiva en el pensamiento jurídico y filosófico posterior.

Varios lustros más tarde y dentro del contexto de una teoría general de la comprensión, Hans-Georg Gadamer reparará en "[e]l significado ejemplar de la hermenéutica jurídica" (Gadamer 1990, p. 330). Esta afirmación de Gadamer descansa, como en Schmitt, en la consideración de que en toda comprensión existe una "tensión" entre reglas y conceptos previos y la "situación" en la que se halla quien comprende. En la medida en que la situación es concreta y singular, la comprensión no puede realizarse por la vía de una subsunción de la situación según unos conceptos universales cuyos contenidos permanezcan incólumes, sino que requiere entender el sentido de las reglas y conceptos también a la luz del sentido de la situación. Gadamer señala, en términos similares a los que emplea Schmitt en Ley y juicio" ${ }^{41}$ : La "aplicación no consiste en la puesta en relación de algo universal previamente dado con la situación particular. El intérprete, que está referido a una tradición, busca aplicarla a sí mismo. Pero esto no quiere decir que el texto tradicional le sea dado como algo universal que deba primero

Ver Schmitt 2011, p. 93. 
entender en sí mismo, para recién luego usarlo en aplicaciones particulares. Más bien, el intérprete no quiere otra cosa que comprender ese universal -el texto-, es decir, comprender lo que dice, qué constituye el sentido y el significado del texto. Para entender eso, es que, precisamente, no puede prescindir de sí mismo y de la situación hermenéutica concreta en la cual él se halla". (Gadamer 1990, p. 329) ${ }^{42}$.

Karl Larenz, quien recoge las consideraciones de Gadamer, indicará que el modo de comprensión jurídico adquiere validez no solo "en un sentido filosófico-jurídico", sino, más ampliamente, en el campo entero "del conocimiento científico-espiritual (geisteswissenschaftlich)". Esa ampliación se justifica porque en la comprensión jurídica se evidencia que el "contenido de sentido (Sinngehalt)" de la regla "debe ser iluminado de manera siempre renovada" y a partir de la consideración de la situación que se trata de comprender (Larenz 1991, pp. 403-404). Incluso alguien eventualmente tan distante de Schmitt como Jacques Derrida, vincula, en "Force de loi", la comprensión jurídica y la comprensión en general, a partir del reconocimiento de una similitud entre la tensión de reglas y casos en la situación jurídica y tal tensión en la situación comprensiva en general $^{43}$.

\section{Referencias bibliográficas}

Adam, Armin (1992), Rekonstruktion des Politischen. Carl Schmitt und Die Krise der Staatlichkeit 1912-1933. Weinheim: Wiley-VCH.

Arendt, Hannah (1992), Lectures on Kant's Political Philosophy. Chicago: The University of Chicago Press.

Bielefeldt, Heiner (1994), Kampf und Entscheidung. Politischer Existentialismus bei Carl Schmitt, Helmuth Plessner und Karl Jaspers. Würzburg: Königshausen \& Neumann.

Derrida, Jacques (1990), "Force de loi: Le 'Fondement mystique de l'autorité"”. Cardozo Law Review 11: 920-1045.

Gadamer, Hans-Georg(1990), Wahrheitund Methode. Grundzüge einerphilosophischen Hermeneutik, en H.-G. Gadamer, Gesammelte Werke. Tübingen: Mohr Siebeck, vol. 1.

Galli, Carlo (1996), Genealogia della politica. Carl Schmitt e la crisi del pensiero politico moderno. Bologna: Il Mulino.

42 "El sentido de la aplicación envuelto en todas las formas de la comprensión ... no consiste en la aplicación posterior a un caso concreto de algo general dado, que sea previamente comprendido en sí mismo" (Gadamer 1990, p. 346).

43 Ver Derrida 1990, pp. 934, 940, 944, 968; para cuando escribió este texto, semejante en varias de sus partes a Gesetz und Urteil de Schmitt, Derrida ya estaba familiarizado con la obra de aquél. Sobre las similitudes y diferencias entre ambos, véase John P. McCormick 2000, p. 1710; 2001, p. 403. 
Habfast, Ulrich (2010), Das normative Nichts. Eine Studie zum Dezionismus in den frühen Schriften Carl Schmitts. Frankfurt: Tesis doctoral.

Halldack, Felix (1912), “Beschprechung der Schrift 'Gesetz und Urteil' von Carl Schmitt”. Kant-Studien 17: 464-467.

Heidegger, Martin (2000), "Die Frage nach der Technik", Vorträge und Aufsätze. Stuttgart: Klett-Cotta, pp. 9-40.

Hofmann, Hasso (2002), Legitimität gegen Legalität. Berlín: Duncker \& Humblot.

Jellinek, Walter (1914), 'Besprechung der Schrift 'Gesetz und Urteil' von Carl Schmitt”, Archiv für öffentliches Recht 23: 296-299.

Kant, Immanuel (1968), Kritik der Urteilskraft, en I. Kant, Akademieausgabe. Berlín: Walter de Gruyter, vol. V.

Kiefer, Lorenz (1990), "Begründung, Dezision und Politische Theologie”, Archiv für Rechts- und Sozialphilosophie 76: 480-482.

Larenz, Karl (1991), Methodenlehre der Rechtswissenschaft. Berlín: Springer.

Marder, Michael (2010), Groundless Existence. The Political Ontology of Carl Schmitt. New York: Continuum.

Maus, Ingeborg (1980), Bürgerliche Rechtstheorie und Faschismus. Zur sozialen Funktion und aktuellen Wirkung der Theorie Carl Schmitts. Munich: Wilhelm Fink.

McCormick, John P. (1997), Carl Schmitt's Critique of Liberalism. Cambridge: Cambridge UP.

(1999), "Three Ways of Thinking 'Critically' about the Law", The American Political Science Review 93: 413-428.

(2000), "Schmittian Positions on Law and Politics?: CLS and Derrida", Cardozo Law Review 21: 1693-1722.

(2001), "Derrida on Law; Or, Poststructuralism Gets Serious", Political Theory 29: 395-423.

Meierhenrich, Jens y Oliver Simons (2016), The Oxford Handbook of Carl Schmitt. Oxford: Oxford UP.

Neumann, Völker (2015), Carl Schmitt als Jurist. Tübingen: Mohr.

Oertmann, Paul (1913), "Besprechung der Schrift 'Gesetz und Urteil' von Carl Schmitt”, Literatursbeilage zur Deutschen Juristenzeitung 18: 817-818.

Ojakangas, Mika (2006), A Philosophy of Concrete Life. Carl Schmitt and the Political Thought of Late Modernity. Bern: Peter Lang.

Rasch, William (2004), "Judgment: The Emergence of Legal Norms", Cultural Critique 57: 93-103.

Scheuerman, William (1999), Carl Schmitt: The End of Law. Oxford: Rowman \& Littlefield.

Schmitt, Carl (1963), Der Begriff des Politischen. Berlín: Duncker \& Humblot.

(1985), Hamlet oder Hekuba. Der Einbruch der Zeit in das Spiel. Stuttgart: Klett-Cotta. 
(1991), Theodor Däublers "Nordlicht”. Drei Studien über die Elemente, den Geist und die Aktualität des Werkes. Berlín: Duncker \& Humblot.

(1993), Über die drei Arten des rechtswissenschaftlichen Denkens. Berlín: Duncker \& Humblot.

(1996a), Politische Theologie. Vier Kapitel zur Lehre von der Souveränität. Berlín: Duncker \& Humblot.

(1996b), Politische Theologie II. Die Legende von der Erledigung jeder Politischen Theologie. Berlín: Duncker \& Humblot.

(1997), Der Nomos der Erde im Völkerrecht des Jus Publicum Europaeum. Berlín: Duncker \& Humblot.

(1998), Politische Romantik. Berlín: Duncker \& Humblot.

(2002), Ex Captivitate Salus. Erfahrungen der Zeit 1945-1947. Berlín: Duncker \& Humblot.

(2003), "Die Lage der europäischen Rechtswissenschaft”, en C. Schmitt, Verfassungsrechtliche Aufsätze aus den Jahren 1924-1954. Berlín: Duncker \& Humblot.

(2009), Gesetz und Urteil. Eine Untersuchung zum Problem der Rechtspraxis. Múnich: Beck.

(2011), Die Tyrannei der Werte. Berlín: Duncker \& Humblot.

(2014), "Staatsethik und pluralistischer Staat", en C. Schmitt, Positionen und Begriffe im Kampf mit Weimar - Genf - Versailles 1923-1939. Berlín: Duncker \& Humblot, pp. 151-165.

(2015), Glossarium. Aufzeichnungen aus den Jahren 1947 bis 1958. Berlín: Duncker \& Humblot.

Schönberger, Christoph (2011), "Werte als Gefahr für das Recht? Carl Schmitt und die Karlsruher Republik”, en C. Schmitt, Die Tyrannei der Werte. Berlín: Duncker \& Humblot, pp. 57-91.

Ulmen, Gary (1991), Politischer Mehrwert. Eine Studie über Max Weber und Carl Schmitt. Weinheim: Wiley-VCH.

Walsmann, (1913), “Besprechung des Buches 'Gesetz und Urteil' von Carl Schmitt”, Rheinische Zeitschrift für Zivil- und Prozessrecht 5: 431-432. 